\title{
Pharmacophore Model Generation of thrombin Inhibitors
}

\author{
Xing Wang ${ }^{1}$, Yanling Zhang ${ }^{1}$, Yuhong Xiang ${ }^{2}$, Zhenzhen Ren ${ }^{1}$, Yanjiang Qiao ${ }^{1 *}$ \\ ${ }^{1}$ School of Chinese Materia Medica, Beijing University of Chinese Medicine, Beijing 100102, China; ${ }^{2}$ Department of \\ Chemistry, Capital Normal University, Beijing 100048, China.
}

Email: yjqiao@263.net

Received 2012

\begin{abstract}
Thrombin, an important factor of clotting system, take part in a variety of physiological actions, such as blood clotting, anticoagulation, thrombosis and fibrinolysis. Inhibiting thrombin is a pivotal and effective step for the prophylaxis of venous and arterial thrombosis, as well as prevent myocardial infarction for high-risk patients. In this study, a three dimensional pharmacophore model was generated for the molecules which are responsible for vasodilation activities targeting thrombin. Ten compounds with known thrombin-inhibiting activity values were selected as training set to generate the hypothesis using GALAHAD program in SYBYL 7.0 software. The best hypothesis comprises five pharmacophore features: four hydrophobes groups and one positively charged group. It has been further validated towards a test set including known activity compounds obtained from Binding Database, the values of effectively active hit $\mathrm{A} \%$ and comprehensive evaluation index CAI are respectively $63.33 \%$ and 2.34 , the pharmacophore was proven to be successful in discriminating active and inactive inhibitors. Furthermore, the pharmacophore model was used as a 3D query to screen TCMD (Version 2009) database and 6 hit compounds of higher predicted activity were the reported cardiovascular activities, which may be useful for further study.
\end{abstract}

Keywords: Cardiovascular Disease; Thrombin; Pharmacophore; Virtual Screening

\section{Introduction}

Thrombin, a "trypsin-like" serine protease protein, is an important factor of clotting system. It take part in a variety of physiological actions, such as blood clotting, anticoagulation, thrombosis and fibrinolysis [1-3]. Moreover, previous studies have demonstrated that thrombin is one of the key factors for cancer developing, such as PAR1, an important type of thrombin, mediates the enhancement effects of thrombin in angiogenesis, invasion and metastasis, growth of cancer [4-6]. These facts have raised great interests to find an effective, safe, and orally available thrombin inhibitors, such as hirudin [7], dabigatran [8], rivaroxaban and apixaban [9], which could be useful anticoagulant drugs for the prophylaxis of venous and arterial thrombosis, as well as prevent myocardial infarction for high-risk patients [10].

In this paper, we have developed a pharmacophore model whose purpose is to identify the critical pharmacophoric features necessary for potent thrombin inhibitors. Further, hypothesis was evaluated by a test set with known activity compounds, the effectively active hit A\% and comprehensive evaluation index CAI were used to assess the model. Finally, the pharmacophore model was used as a 3D query to screen TCMD (Version 2009) database, 52 compounds were hit and the 6 hit compounds of higher predicted activity were the reported cardiovascular activities, which may be useful for further study.

\section{Materials and Methods}

\subsection{Compounds and biological data}

Compounds $1 \sim 30$, which can inhibit thrombin, were taken from the literatures [11-13] and served as the database in the pharmacophore modeling. The structures and inhibitory activities are listed in Figure 1. The chemical structures were drawn in ISIS-Draw software and saved in SYBYL mol2 format, then all the 2D structures were converted to 3D structures in SYBYL 7.0 software.

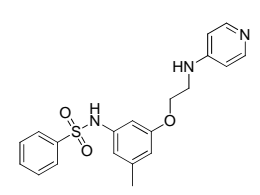

Compound 1

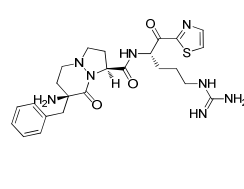

Compound 2

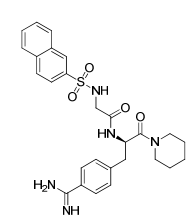

Compound 3 


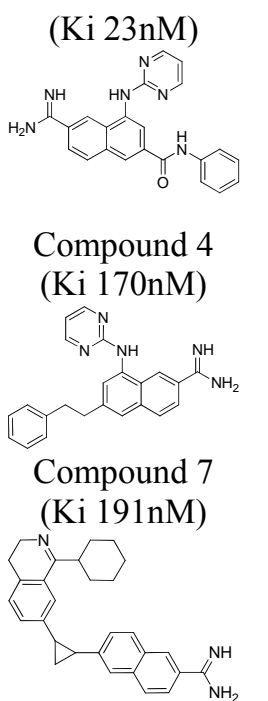

Compound 10

(Ki 1136nM)

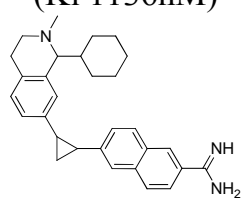

Compound 13

(Ki 996nM)<smiles>NC(N)c1ccc2cc(/C=C/c3ccccc3)ccc2c1</smiles>

Compound 16

(Ki 5269nM)

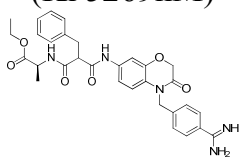

Compound 19

(Ki 21300nM)

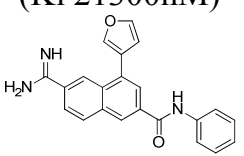

Compound 22

(Ki 132nM)

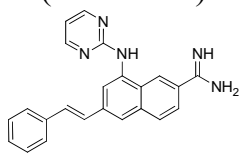

Compound 25

(Ki 395nM)<smiles>N=C(N)c1ccc(Cc2ccccc2)cc1</smiles>

Compound 28

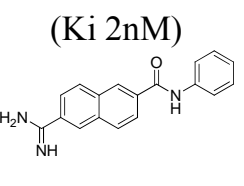

Compound 5

(Ki 5640nM)

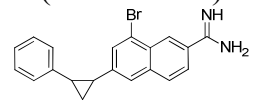

( $\mathrm{Ki} 7 \mathrm{nM})$

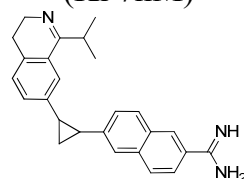

Compound 6

(Ki 2139nM)

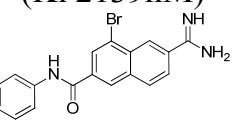

Compound 8

Compound 9

(Ki 503nM)
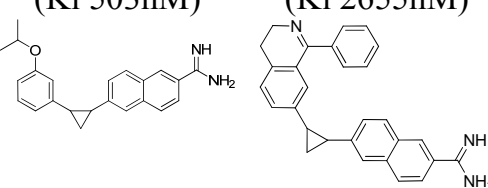

Compound 11

(Ki 1099nM)

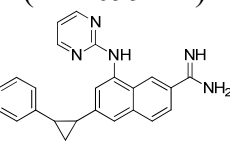

Compound 12
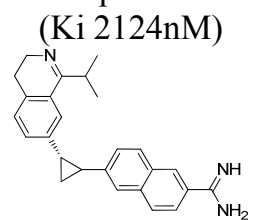

Compound 14 Compound 15

(Ki 48nM)

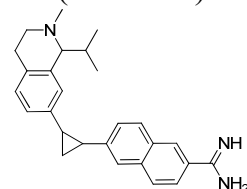

Compound 17

(Ki 1796nM)

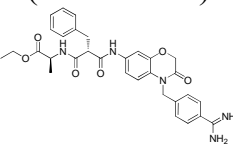

Compound 20

(Ki 20600nM)

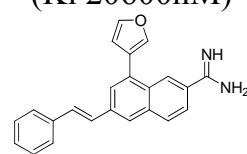

Compound 23

(Ki 237nM)

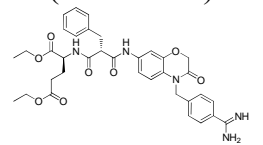

Compound 26

(Ki 23300nM)

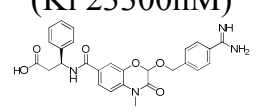

(Ki 1790nM)

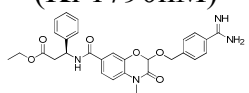

Compound 18

(Ki 3700nM)

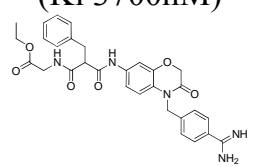

Compound 21

(Ki 12100nM)

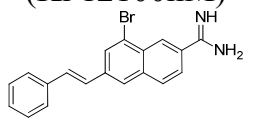

Compound 24

(Ki 920nM)

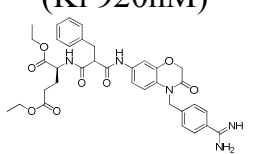

Compound 27

(Ki 21600nM)

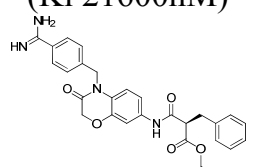

Compound 29 Compound 30
(Ki 1890nM)

(Ki 14900nM)

(Ki 16300nM)

Figure 1. Chemical structure of thrombin inhibitors.

\subsection{Modeling tool}

All the pharmacophore modeling calculations were carried out by using the GALAHAD module implemented in SYBYL 7.0 (Tripos Inc.) software, the UNITY module was used to perform a flex search for the potential inhibitors. All the tasks were running on Xeon Intel(R) X5460 CPU $3.16 \mathrm{GHz}$, RAM Memory 12 GB under the Windows XP system.

\subsection{Pharmacophore studies}

GALAHAD (Genetic Algorithm with Linear Assignment of Hypermolecular Alignment of Datasets) uses Tripos' proprietary technology [14-15] to generate pharmacophore hypotheses and alignments from sets of ligand molecules that bind at a common target site. Before establishing the model, all the structures of ligands were checked, hydrogen atoms were added and a minimization procedure was implemented using the MMFF94 force-field. GALAHAD was run for 130 generations with a population size of 100 . The rest of the parameters were set as default values. Only models with 8 ligands with contribution to the consensus feature were considered. The generated models were evaluated by a test database containing 111 compounds, including 30 active compounds and 81 non-active compounds.

\subsection{Virtual Screening}

The pharmacophore models built within the Catalyst software can be used as queries for 3D database screening. Virtual screening of such databases can serve two main purposes: first, validating the quality of the generated pharmacophore models by selective detection of compounds with known thrombin inhibitory activity, and second, finding novel, potential leads suitable for further development. In this study, the best pharmacophore model was used to search TCMD (Version 2009) to quest for the potential inhibitors of thrombin from traditional Chinese medicine.

\section{Results and Discussion}

\subsection{Generation of pharmacophore hypotheses}

GALAHAD models were derived by using ten active ligands as a training set [compounds 4-6, 8, 9, 15, 17, 18, 25, 28], 20 pharmacophore models were derived by using the training set after GALAHAD run. All the 20 models were evaluated successively by the test database constructed previously. Table 3 shows the predictable results of the test database for models with all the eleven ligands with contribution to the consensus feature. 
In order to intuitively understand the meaning of indicators used to evaluate the performance of the models, the schematic diagram was listed (Figure 2) and the parameter values for each pharmacophore model generated were listed in Table 1. D is for the total number of compounds in test database and A represents the number of active compounds. Ht is the total number of hit compounds from test database and Ha represents the number of active hit compounds from test database, A\% represents the ability to identify active compounds from test database, $\mathrm{Y} \%$ represents the proportion of active compounds in the hit compounds. $\mathrm{N}$, the index of effective identification, is used to evaluate the ability of the models to identify active compounds from the non-active compounds. CAI, a comprehensive evaluation index, is used to identify the best pharmacophore model. Higher value of CAI is considered to be the better model. In this study, MODEL_016, with the highest value of CAI was considered to be the best model.

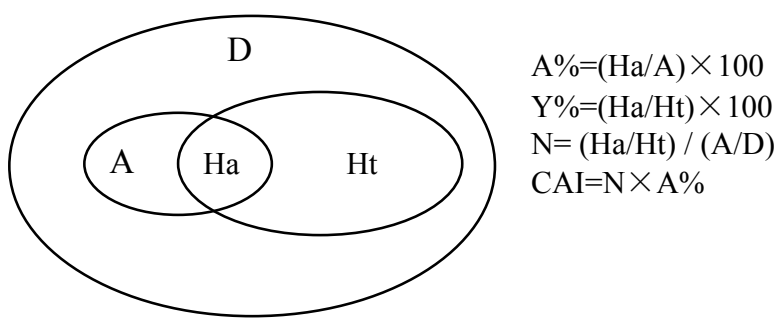

Figure 2. The schematic diagram of indicators used to evaluate the pharmacophore model

Table 1. The parameter values for each pharmacophore model

\begin{tabular}{lcccccc}
\hline & Ht & Ha & A\% & Y\% & N & CAI \\
\hline MODEL_01 & 12 & 12 & 40.00 & 100.00 & 3.70 & 1.48 \\
MODEL_02 & 17 & 17 & 56.67 & 100.00 & 3.70 & 2.10 \\
MODEL_03 & 14 & 14 & 46.67 & 100.00 & 3.70 & 1.73 \\
MODEL_04 & 19 & 19 & 63.33 & 100.00 & 3.70 & 2.34 \\
MODEL_05 & 9 & 9 & 30.00 & 100.00 & 3.70 & 1.11 \\
MODEL_06 & 29 & 21 & 70.00 & 72.41 & 2.68 & 1.88 \\
MODEL_07 & 17 & 17 & 56.67 & 100.00 & 3.70 & 2.10 \\
MODEL_08 & 16 & 16 & 53.33 & 100.00 & 3.70 & 1.97 \\
MODEL_09 & 6 & 6 & 20.00 & 100.00 & 3.70 & 0.74 \\
MODEL_10 & 19 & 19 & 63.33 & 100.00 & 3.70 & 2.34 \\
MODEL_11 & 17 & 17 & 56.67 & 100.00 & 3.70 & 2.10 \\
MODEL_12 & 19 & 19 & 63.33 & 100.00 & 3.70 & 2.34 \\
MODEL_13 & 19 & 19 & 63.33 & 100.00 & 3.70 & 2.34 \\
MODEL_14 & 19 & 19 & 63.33 & 100.00 & 3.70 & 2.34 \\
\hline
\end{tabular}

\begin{tabular}{lllllll}
\hline MODEL_15 & 14 & 14 & 46.67 & 100.00 & 3.70 & 1.73 \\
MODEL_16 & $\mathbf{1 9}$ & $\mathbf{1 9}$ & $\mathbf{6 3 . 3 3}$ & $\mathbf{1 0 0 . 0 0}$ & $\mathbf{3 . 7 0}$ & $\mathbf{2 . 3 4}$ \\
MODEL_17 & 11 & 11 & 36.67 & 100.00 & 3.70 & 1.36 \\
MODEL_18 & 17 & 17 & 56.67 & 100.00 & 3.70 & 2.10 \\
MODEL_19 & 14 & 14 & 46.67 & 100.00 & 3.70 & 1.73 \\
MODEL_20 & 13 & 13 & 43.33 & 100.00 & 3.70 & 1.60 \\
\hline
\end{tabular}

MODEL_016 is displayed in Figure 3, cyan and red spheres indicate hydrophobes group and positively charged charged group, respectively. MODEL_016 includes five pharmacophore features: four hydrophobes groups and one positively charged group.

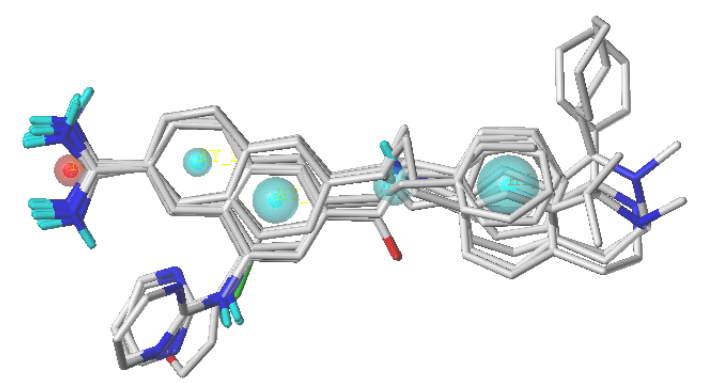

Figure 3. Pharmacophore MODEL_016 and molecular alignment of the compounds used to elaborate the model.

\subsection{Virtual Screening}

Pharmacophore MODEL_016 was used to screen TCMD (Version 2009). TCMD screening yielded a hit list of 52 compounds and the 6 hit compounds of higher predicted activity (Bufotoxin, Cyclovirobuxine, Cyclovirobuxine D, Leurosine, Methoxyadiantifoline and Thaliadanine) were the reported cardiovascular activities, such as vasodilator, anti-arrhythmic, cardiac, coronary vasodilator and anti-hypertension, et al. Cyclovirobuxine mapped to MODEL_016 is showed in Fig 4.

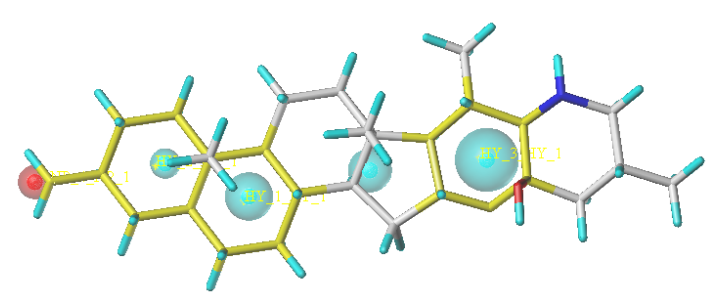

Figure 4. MODEL_016 mapped with Cyclovirobuxine

\section{Conclusions and Future Work}

In this study, we have built the pharmacophore model of thrombin inhibitors using GALAHAD module implemented in SYBYL 7.0 software. The optimal pharmacophore model contains 5 pharmacophore features: four 
hydrophobes group and one positively charged charged group. The external validation shows that the values of effectively active hit $\mathrm{A} \%$ and comprehensive evaluation index CAI are respectively $63.33 \%$ and 2.34 , which can prove that the pharmacophore model is reliable and available. The virtual screen results of TCMD (Version 2009) database shows that the pharmacophore model has the ability of determining vasoactive compound related to thrombin, and the validation experiments needs to be further studied.

\section{Acknowledgements}

This work is financially supported by the Foundation of National Natural Science Foundation of China (No. 81173522) and National Key Technology R\&D Program (No. 2008BAI51B01) in Beijing University of Chinese Medicine.

\section{REFERENCES}

[1] Sambrano GR, Weiss EJ, Zheng YW, et al. "Role of thrombin signalling in platelets in haemostasis and thrombosis". Nature, Vol. 413, No. 6, 2011, pp. 74-78. doi:10.1038/35092573

[2] H LUO, Y LIANG. "Thrombin-activatable fibrinolysis inhibitor: a new modulator linking coagulation and fibrinolysis". Basic Medical Sciences and Clinics, Vol. 23, No. 5, 2003, pp. 484-489. doi:6325(2003)05-0484-06

[3] Weitz JI. "Factor Xa and thrombin as targets for new oral anticoagulants". Thrombosis Research, Vol. 127, No. 2, 2011, pp. S5-S12. doi:10.1016/S0049-3848(10)70147-X

[4] Crawley JT, Zanardelli S, Chion CK, et al. "The central role of thrombin in hemostasis". Journal of Thrombosis and Haemostasis, Vol. 5, Sup. s1, 2007,pp. 95-101. doi:10.1111/j.1538-7836.2007.02500.x

[5] Nierodzik ML,Karpatkin S. "Thrombin induces tumor growth, metastasis, and angiogenesis: Evidence for a thrombin-regulated dormant tumor phenotype". Cancer Cell, vol.10,No.5,2006, pp.355-362. doi.org/10.1016/j.ccr.2006.10.002

[6] Bajzar L, Chan AK, Massicotte MP, et al. "Thrombosis in children with malignancy". Current Opinion in Pediatrics,vol.18,No.1, $2006 \quad$,pp.1-9. doi:10.1097/01.mop.0000193270.09001.ea
[7] Eriksson BI, Quinlan DJ, Eikelboom JW. "Novel Oral Factor Xa and Thrombin Inhibitors in the Management of Thromboembolism". Annual Review of Medicine, vol.62,2011,pp. 41-57.

doi:10.1146/annurev-med-062209-095159

[8] Bendel SD, Bona R, Baker WL. "Dabigatran: an oral direct thrombin inhibitor for use in atrial fibrillation". Advances in Therapy,vol.28,No.6,2011,pp.460-472. doi:10.1007/s12325-011-0025-1

[9] A. Kobsar , J. Koessler , L. Kehrer,etal. "The thrombin inhibitors hirudin and Refludan ${ }^{\circledR}$ activate the soluble guanylyl cyclase and the cGMP pathway in washed human platelets". ThrombosisandHaemostasis.Vol.107.No.3,2012,pp.521-529. doi:10.1160/TH11-07-0461

[10] Steinmetzer T, Sturzebecher J. "Progress in the Development of Synthetic Thrombin Inhibitors as New Orally Active Anticoagulants".Current Medicinal Chemistry,Vol.11,No.17, doi: $10.2174 / 0929867043364540$

[11] Santosh P, Gregory A. L., Julie E. P. "Conformation Mining: An Algorithm for Finding Biologically Relevant Conformations”. J. Med. Chem., Vol. 48, No.9, 2005, pp. 3313-3318. doi:10.1021/jm0490661.

[12] Milan Bruncko, William J. McClellan, Michael D. Wendt, et al. "Naphthamidine urokinase plasminogen activator inhibitors with improved pharmacokinetic properties". Bioorganic \& Medicinal Chemistry Letters, Vol.15,No.1, 2005, pp. 93-98. doi:10.1016/i.bmcl.2004.10.026

[13] Petra Štefanic Anderluh, Marko Anderluh, Janez Ilaš. "Toward a Novel Class of Antithrombotic Compounds with Dual Function. Discovery of 1,4-Benzoxazin-3(4H)-one Derivatives Possessing Thrombin Inhibitory and Fibrinogen Receptor Antagonistic Activities", J. Med. Chem., 2005, 48 (9), pp 3110-3113. doi:10.1021/jm048984g

[14] Richmond, N.J., Abram, C.A., Wolohan, P.R., et al. "GALAHAD: 1 Pharmacophore Identification by Hypermolecular Alignment of Ligands in 3D". J COMPUT AID MOL DES, Vol. 20, No. 9, 2006, pp. 567-587. doi:10.1007/s10822-006-9082-y.

[15] N.J. Richmond, P.Willett, R.D. Clark. "Alignment of three-dimensionalmolecules using an image recognition algorithm". J.Mol.Graph.Model. Vol. 23, No. 2, 2004, pp. 199-209. doi:10.1016/j.bbr.2011.03.031. 